\title{
Performance of exchange-correlation functionals on describing ground state geometries and excitations of Alizarin Red S: Effect of complexation and degree of deprotonation
}

$$
\text { Péter Pál Fehér }{ }^{1} \text {, Mihály Purgel }{ }^{1,2} \text { and Ferenc Joo }{ }^{1,2}
$$

${ }^{1}$ Department of Physical Chemistry, University of Debrecen, H-4032 Debrecen, Egyetem tér

$$
\text { 1., Hungary }
$$

${ }^{2}$ MTA-DE Homogeneous Catalysis and Reaction Mechanisms Research Group, H-4032 Debrecen, Egyetem tér 1., Hungary

\section{CORRESPONDING AUTHORS:}

*Mihály Purgel, MTA-DE Homogeneous Catalysis and Reaction Mechanisms Research Group, H-4032 Debrecen, Egyetem tér 1., Hungary, Tel.: +36 52-512-900/22314, E-mail: purgel.mihaly@science.unideb.hu

\begin{abstract}
$\underline{\text { Abstract }}$
Ground state optimizations and excited state calculations were performed to analyze the possible anionic forms of Alizarin Red $\mathrm{S}$ (ulphonated) and its $\mathrm{ML}_{2}$ type metal complexes formed with palladium(II). Six functionals have been tested (B3LYP, M06-2X, M06, BH\&HLYP, PBE0, LC- $w$ PBE, and CAM-B3LYP) with two basis sets $(6-311+\mathrm{g}(\mathrm{d}, \mathrm{p})$ and TZVP). The relative errors of these functionals in reproducing the experimental UV-Vis absorption wavelengths are reported for the energetically lowest lying isomers. It was found that the degree of deprotonation affects several functionals in a systematic way. M06 and CAM-B3LYP $x c$-functionals gave the best estimates according to the average relative errors. These two functionals were then used to explore the coordination mode (hydroxy-keto or catechol) of the palladium(II) complexes and the effect of (de)protonation.
\end{abstract}

Keywords: DFT, TDDFT, Alizarin Red S, ARS, palladium 


\section{$\underline{\text { Introduction }}$}

Alizarin (1,2-dihydroxy-9,10-anthraquinone, Alizarin Red) is widely used but still investigated molecule. In particular, its clinical and industrial applications (textile dyes, biolabeling, optical device manufacturing, etc.) are major topics of recent researches [1-8]. It is also a popular $\mathrm{pH}$ indicator with color changes related to the (de)protonation of the $-\mathrm{OH}$ group in the $\mathrm{C} 2$ position. Interactions of different metal ions with Alizarin are used for a wide range of analytical processes such as trace metal extraction or quantification and also for biological application such as calcium ion assay [1-3, 7]. Alizarin Red has low water solubility therefore in aqueous solution its sulphonated derivate (Alizarin Red S, ARS) is used.

Many experimental and theoretical works deal with the optical and excited state properties and/or the tautomeric forms of alizarin and its ionized species [9-15]. However, most publications concerned specifically with sulphonated alizarine (ARS) are experimental studies and no calculations were made on its spectral properties [7, 16-19]. There are articles and reviews dedicated to the testing the TDDFT performance of different $x c$-functionals which include anthraquinone derivatives [9-12, 20, 21]. In these works the emphasis is mostly on giving a general description on the performance of the selected functionals so choosing the appropriate one for a particular molecular system is not straightforward. Although they consider a wide variety of compounds, the degree of deprotonation is rarely taken into account $[10,11]$.

According to previous works PBE0 and CAM-B3LYP functionals showed the best performance for calculating excitation energies. With the current state of the art we are able to improve and extend the previous results $[10,11]$ using the new IEF-PCM algorithm [22] and the M06 class-functionals [23]. We are mainly interested in how the performance of the chosen functionals change if we "start removing protons" from the ARS and also whether the best performing functionals can reliably describe ARS complexes, too. Another difference between this work and the previous results is that in the literature several TDDFT studies were carried out in a way that they calculated excited states with different functionals in a single point fashion on structures optimized using a chosen level of theory [9]. Our treatment includes both ground state DFT and TDDFT calculations with the same selected functionals.

In the second part of the present work we explore the complex formation of ARS with palladium(II). Studies on alizarin complexes are concerned with a wide variety of metal ions [24-26], but the following conclusion can be made: in acidic medium the metal ions form 
complexes through the oxygen atom of the carbonyl group and the oxygen atom of the phenolate group at $\mathrm{C} 1$ position called the hydroxy-keto coordination. Contrarily in alkaline medium there is a catechol coordination type involving the two phenolate oxygens in ortho positions.

Joó et al. found that the complexation with palladium(II) yields a powerful catalyst for hydrogenation reactions of lipids in aqueous solution. However, this Pd-complex needs to be pre-hydrogenated in order to avoid long induction periods when used in reactions. During this process formation of several products has been observed and each of them gives a specific UV-Vis spectrum [27]. The Pd-ARS complex was first prepared by Bulatov et al. and they suggested a hydroxy-keto coordination based on IR spectroscopic measurements [28]. There is an inconsistency between the UV-Vis absorption wavelengths reported in these two papers which may be due to the uncertainty in the $\mathrm{pH}$ of the Pd-ARS solutions investigated.

As ARS and also its complexes are water soluble the $\mathrm{pH}$ of the media cannot be neglected and in order to properly describe the given molecular system we need a computational method that gives consistent results. Because this current paper may initiate further experimental and theoretical research on Pd-ARS complexes (for example a study about the yet unknown structures of the hydrogenated derivatives reported by Joó et al.) selecting a reliable level of theory for these calculations is crucial. 


\section{Calculation details}

All calculations were performed using the C.01 revision of Gaussian 09 software package [22]. The results presented in this paper were obtained by performing multi step jobs. The first step was full ground state geometry optimizations followed by frequency calculations. In the second step excited state calculations were carried out using timedependent density functional theory (TDDFT). The open source GaussSum software was then used to generate UV-Vis spectra from the calculated data [29]. After examining the obtained $\mathrm{UV}-\mathrm{Vis}$ spectra it has been found that calculating the first 30 singlet excited states is enough to reproduce the peak shapes and wavelength range observed in the experimental spectra. Gaussian09's default IEF-PCM solvent model was used in every case to account for the effect of water [30]. The reported relative energies in this work are derived from the calculated zeropoint corrected electronic energies of the corresponding structures.

In this work six plus one $x c$-functionals have been tested: (1) B3LYP [31], (2) M062X [23], (3) M06 [23], (4) BH\&HLYP [32], (5) PBE0 [33], (6) LC-wPBE [34, 35], and CAM-B3LYP [36] but for this last functional B3LYP/6-311+g(d,p) optimized geometries were used for single point TDDFT calculations using the same basis set. The first four functionals are hybrid type with constant amount of exact HF exchange often referred in the literature as global hybrids. LC-wPBE and CAM-B3LYP are long range corrected (or rangeseparated) hybrids where the amount of exact exchange grows with increasing interelectronic distance to make them more accurate for transitions to higher lying orbitals [9, 22]. Long range corrected hybrids offer the most improvement over global hybrids when dealing with large molecular systems, such as conjugated polymers, weakly bound complexes or calculating charge-transfer states.

In addition to varying functionals two different basis sets for ARS calculations have been tested: (1) $6-311+\mathrm{g}(\mathrm{d}, \mathrm{p})$ is a Pople-type triple- $\zeta$ basis with one set of diffuse and polarization functions [37], (2) TZVP which is also a triple- $\zeta$ basis [38] and about the same size as the first one so a little difference should be expected between these two .

For the palladium(II) complexes we used the following user-specified basis: CRENBL basis with effective core potential (ECP) on the metal [39] and TZVP basis set on the rest of the atoms. Computational results in the literature show very good agreement with experimental results when CRENBL ECP is used on heavy (metal) atoms especially for spectroscopic applications [40]. 


\section{$\underline{\text { Results }}$}

In the first part of this section we provide the results of the ground state calculations (geometry optimizations). In the second part the relative errors of different $x c$-functionals in calculating the UV-Vis absorption wavelengths of the structures calculated in the first part are presented. In the third part we show the results obtained for palladium(II)-ARS $\mathrm{ML}_{2}$ type complexes.

Although our goal was to give the most complete description of the different forms of ARS in aqueous medium, no neutral molecules were taken into account as it was assumed that the sulfo group is deprotonated in every structure (Fig. 1). Among the dianionic forms we only considered cases where intramolecular hydrogen bonding is possible (Fig. 2).

The reason behind this thorough investigation was the change in solvent compared to previous articles dealing with alizarin from a theoretical point of view. In water there is a complicated dynamic equilibrium between the tautomers and also, one must take into consideration the deprotonation of one or both phenol groups.

Fig. 1 shows the results of the ground state calculations for the monoanionic forms of ARS. The listing order of the isomers is of no importance but the relative energies and the general trend - the shape of the lines - are. The third and seventh points show interesting behavior and those points indeed are the main difference between the tested functionals. In the case of PBE0, LC- $w$ PBE and M06-2X functional 3 transforms spontaneously into 2 during geometry optimization and the same occurs for 7 (to 6) when using LC- $w$ PBE and M06-2X. Isomer 8 has a more elaborate conjugated $\pi$ system of electrons that cannot be represented with just placing double bonds in the structural formula and it is reflected by its highest relative energy. Fig. 3 shows the basis set effect by taking the difference between the relative energy plots given by the two basis sets. The difference for the dianionic forms is negligible (between 1.08 and $-3.64 \mathrm{~kJ} / \mathrm{mol}$ ), but in the monoanionic case much greater effect is observed. To emphasize this, the vertical axis two of the plots in Fig. 3 has the same scale. The big differences show that B3LYP and PBE0 change behavior if we switch from the Pople-basis to TZVP, transforming 3 to 2 and 7 to 6 , respectively.

These results should not tell us that some functionals are "bad" or inaccurate, but rather which ones are consistent when describing the same process (quinonic $\mathrm{OH}$ to catechol $\mathrm{OH}$ occurs during both 3 to 2 and 7 to 6 conversions) or changing basis sets. It can be said that M06, BH\&HLYP, M06-2X and LC-wPBE give consistent results with the first two 
finding 3 and 7 on the potential energy surface and the last two giving spontaneous conversions to 2 and 6.

Fig. 2 shows the results of geometry optimizations on the dianionic forms of ARS. No spontaneous transition occurred between the calculated structures and also no basis set effect was observed. The first four structures have the proton on one of the catechol oxygen atoms and the tested functionals give similar results for their energies with only M06-2x and LC$w$ PBE deviating slightly from the others. However, for the last structure - where the proton is on one of the quinonic oxygen atoms - significant scattering can be observed among the energy calculated by the different functionals.

ARS, similarly to alizarin is a bidentate ligand in complex forming reactions. 2' and 3' can be thought of as the two extremity of an ML type complex where a proton is placed on an oxygen atom instead of a metal ion somewhere between the two coordinating oxygen atoms. This thinking can be applied to 4' and 5' which structures resemble a hydroxy-keto coordinated complex. For catechol coordination these two extremities are close in energy $(<$ $10 \mathrm{~kJ} / \mathrm{mol}$ ) and therefore it can be said that this coordination mode is balanced. However, for hydroxy-keto coordination the energy difference between 4' and 5' is much greater (more than $20 \mathrm{~kJ} / \mathrm{mol}$ ), 4' being clearly lower in energy so a distorted bonding can be expected where the metal prefers one of the coordinating oxygen atoms over the other which makes hydroxy-keto coordination less favorable. There are significant differences in the hydrogen bond distances in the six-membered rings of 4' and 5' compared to the five-membered rings of 2' and 3' which also support this train of thought (Table I).

The main transition in the experimental UV-visible absorption spectra of the different forms of alizarin is a $\pi-\pi^{*}$ type excitation occurring between the bonding and antibonding MOs of the anthraquinone ring. As the molecule deprotonates, the wavelength shifts from $425 \mathrm{~nm}$ to the $510 \mathrm{~nm}$ region. The calculations show that this transition comes from only the aforementioned electronic excitation in contrast to the peak observed below $300 \mathrm{~nm}$ which includes most of the 30 calculated transitions. In the experimental spectra a third peak can be observed between $\mathrm{pH} \approx 6-10$ where the presence of the dianionic form is expected.

There are two ways in which we can compare the performances of the functionals: (1) finding the spectra that is the most similar to the experimental one and taking the corresponding molecular structure (2) comparing the calculated spectra of the energetically lowest lying structure to the experimental spectra. The first approach can only give a vague impression because in excitation energy calculations the error is bigger than in ground state energy calculations and as it can be seen from our results several functionals don't match the 
spectrum most similar to experimental results to the lowest energy structure. This is the reason why in Table II BH\&HLYP and LC- $w$ PBE does not give a second peak between 300 and $400 \mathrm{~nm}$ in the dianionic case as we used (2) to compare the functionals. However, in the case of BH\&HLYP the spectrum of 5' have excitations at 534 and $320 \mathrm{~nm}$ and those values are almost the same as experimental ones at 510 and $326 \mathrm{~nm}$. The only problem with $5^{\prime}$ is that it is the highest energy structure among the dianionic forms calculated by BH\&HLYP. The mentioned two functionals overestimate the excitation energies and that is the reason for the middle peak of the spectrum to appear below $300 \mathrm{~nm}$.

Experimental excitation wavelengths are obtained from Ref. 19. The relative errors of different functionals calculated with the following formula are presented in Table II:

$$
\text { relative error }=\frac{\lambda_{\text {calc }}-\lambda_{\max , \exp }}{\lambda_{\max , \exp }}
$$

The results show that B3LYP, M06 and PBE0 overestimate while CAM-B3LYP, M06-2X, BH\&HLYP and LC- $w$ PBE underestimate the experimental wavelengths based on the overall average of the calculated errors. The best estimates are given by M06 and CAMB3LYP with 5.0\% and $-5.7 \%$ mean relative errors respectively, M06 being the only functional which never exceeds $10 \%$ relative error within the three ionic forms. The degree of deprotonation has the biggest effect on the performance of LC-wPBE, BH\&HLYP, M06-2X functionals, especially for M06-2X, where the fully deprotonated species is very well described (below 3\%), but for the monoanionic form it gave more than $15 \%$ relative error (which is in absolute error more than $65 \mathrm{~nm}$ ).

Complexes of ARS formed with palladium(II) are of square planar geometry due to the $\mathrm{d}^{8}$ electron configuration. In case of ARS as a bidentate ligand two coordination modes are possible, one is the catechol - where the deprotonated hydroxyl groups at $\mathrm{C} 1$ and $\mathrm{C} 2$ positions coordinate - the other is the hydroxy-keto coordination with a quinonic oxygen and a deprotonated hydroxyl group at the $\mathrm{C} 1$ position. Depending on the orientation of the ligand the complex can belong in either a $\mathrm{C} 2 \mathrm{v}$ or $\mathrm{C} 2 \mathrm{~h}$ point group.

Fig. 4 and 5 show the considered $\left[\mathrm{Pd}^{\mathrm{II}}(\mathrm{HARS})_{2}\right]^{2-}$ and $\left[\mathrm{Pd}^{\mathrm{II}}(\mathrm{ARS})_{2}\right]^{4-}$ structures and their calculated relative zero point corrected electronic energies. Among complexes with dianionic ligands hydroxy-keto coordination is a lot more favorable than catechol coordination which order is reversed after removing an additional proton from the ligands. This behavior can be explained by looking at the drawn structures of cathecol coordinated 
$\left[\mathrm{Pd}^{\mathrm{II}}(\mathrm{HARS})_{2}\right]^{2-}$ complexes where to keep the conjugated $\pi$ system the quinonic subunit breaks and one of the catechol hydroxyl groups (both if we consider the resonance forms) gains carbonyl character which contributes a weaker bond to the palladium. In the deprotonated case, both of the coordinating oxygens in the catechol coordination are phenolate type and therefore can bond the metal stronger. These results are in good correspondence with what was concluded from the ARS calculations. This tells us that at lower $\mathrm{pH}$ values formation of hydroxy-keto, at higher $\mathrm{pH}$ values catechol coordinated complexes are expected. In the present work the transition (rotation of ligands or dissociation and reformation of the complexes) between the two forms was not taken into account, but considering that the relative energy of $\mathrm{c5}^{\prime}$ (mixed coordination complex) is between the "pure" states - it should not be considered impossible. The effect of symmetry (point group) has been found to have only a slight effect on the relative energies of the complexes.

The results of the TDDFT calculations on the Pd-complexes are more difficult to discuss than in the case of ARS. The two experimental results presented in Refs. 23 and 24 are rather different. Bulatov et al. report only one peak with $\lambda_{\max }$ of $530 \mathrm{~nm}$ at $\mathrm{pH}=4.01$ and $630 \mathrm{~nm}$ at $\mathrm{pH}=6.86$ and they attribute it to c2. In the work of Joó et al. the UV-visible spectra of the possibly c1 complex is shown during a pre-hydrogenation process where the form " $\mathrm{A}$ " - which we deal with in the present work - exhibits peaks at 333 and $520 \mathrm{~nm}$. They worked with a $\mathrm{pH}=6.5$ solution.

We compared the calculated spectra to the spectra given in Ref. 24, and got the closest match in peak shapes for c1' with both CAM-B3LYP and M06. c1' is C2h type has essentially the same spectra as c2' with $\mathrm{C} 2 \mathrm{v}$ symmetry and it can be said that the effect of symmetry on every other calculated UV-Vis spectrum is also negligible. All the other calculated spectra have peaks between 400 and $500 \mathrm{~nm}$ which are not present in the experimental spectrum and have very different overall shapes. The problem with interpreting these results is that $\mathrm{cl}^{\prime}$ is not the lowest energy $\left[\mathrm{Pd}^{\mathrm{II}}(\mathrm{ARS})_{2}\right]^{4-}$ structure.

According to the results of Bulatov et al., the deprotonation of the complex is accompanied by a bathochromic shift of the reported absorption maximum. This behavior is observed in the calculated spectra of $\mathrm{c} 1$ and $\mathrm{c} 1$ ' (also for $\mathrm{c} 2$ and $\mathrm{c} 2$ '), but in the case of the catechol coordination (c3-c3' and c5-c4') a hypsochromic shift is observed instead. These results and the good agreement of calculated peak shapes of $\mathrm{cl}^{\prime}$ ' with experimental spectra indicate the presence of hydroxy-keto coordinated complexes. However, the calculated energies suggest that catechol coordination should take place at higher $\mathrm{pH}$ values, which contradiction cannot be lifted at these levels of calculations. 
Although the general conclusions so far were the same, there are a few important differences between the two functionals: (1) TDDFT calculations with CAM-B3LYP give higher excitation energies than M06. It can be seen from the first peaks near $300 \mathrm{~nm}$ that there is about a $50 \mathrm{~nm}$ difference between the two functionals. This can also be observed in the ARS calculations (Table II). (2) For the majority of the structures oscillator strengths calculated with CAM-B3LYP are significantly higher. The spectra presented in this article have different half-width for the two functionals to give similar peak shapes. (3) The peaks observed at higher wavelengths consist of different numbers of excitations. ARS calculations give one low-energy excitation with mainly $\mathrm{HOMO} \rightarrow$ LUMO character therefore the evaluation of the calculated results was much simpler. In the spectra of c1' - which resemble the experimental spectrum - there is one such excitation calculated with CAM-B3LYP, while the peak in the M06 spectrum consists of two lines.

The molecular orbitals involved in the lowest lying excited state (CAM-B3LYP) of c1' with significant $(>0.1)$ oscillator strength are shown in Fig. 8. For M06 there are two such excited states with major contributions from these MOs. The one at $617 \mathrm{~nm}$ has $92 \%$ HOMO $\rightarrow$ LUMO the other at $537 \mathrm{~nm}$ has $94 \% \mathrm{H}-1 \rightarrow \mathrm{L}+1$ contribution. Both of these excitations have a charge-transfer character. In the case of CAM-B3LYP the main low energy excitation is a mixture between the states calculated with M06. It has wavelength at $543 \mathrm{~nm}$ and includes $65 \% \mathrm{HOMO} \rightarrow$ LUMO and 33\% H-1 $\rightarrow$ L+1 excitations with oscillator strength $(0.4438)$ near the sum of those of the previous M06 states $(0.1937$ and 0.1055$)$. 


\section{$\underline{\text { Conclusion }}$}

We have performed DFT calculations using six $x c$-functionals for the anionic forms of Alizarin Red S. From the relative energies of the mono- and dianionic forms assumptions were made for the coordination mode of the palladium(II) complexes of ARS in acidic and alkaline media. The calculations on the metal complex were in good agreement with these presumptions. Energetically the catechol coordination mode is favored where the ligand is fully deprotonated. In the case of dianionic ligands - which complex is expected to form in a slightly acidic medium - hydroxy-keto coordination is preferred. Time-dependent density functional theory (TDDFT) calculations were carried out to verify these results but the shape of the simulated absorption of the catechol isomers are significantly different in comparison with the experimental spectra. However, the calculated spectra of the hydroxy-keto coordinated complexes gave peak shapes similar to the experimental ones and reproduced the experimentally observed bathochromic shift when the $\mathrm{pH}$ of the solution is raised.

Testing the functionals showed that the main difference between the considered $x c$ functionals is in the excited state (TDDFT) calculations. The results of the ground state calculations - which are the geometries and relative energies of the tautomers - show more or less the same relative energy order for every functional. The only difference is that there are some functionals which cannot identify isomers 3 and 7 as true minima on the potential energy surface. The change in basis set only affected the B3LYP and PBE0 functionals noticeably; the rest of the functionals gave consistent results. Performance analysis was carried out on the calculated excitation wavelengths. Functionals with more than $10 \%$ average relative errors (B3LYP, BH\&HLYP and LC- $w$ PBE) fail to match the calculated spectra of the energetically lowest lying isomer to the experimental ones in case of one or more of the three anionic forms, and therefore those are not reliable for TDDFT calculations on molecules similar to ARS. The degree of deprotonation affects CAM-B3LYP, M06-2X, BH\&HLYP and LC- $w$ PBE functionals in a systematic way where going from the monoanionic species to the fully deprotonated form the relative errors get smaller and smaller. The performance of B3LYP was improved by using the CAM-B3LYP $x c$-functional for TDDFT. Best estimates to the experimental wavelengths were given by M06 and the CAM-B3LYP "augmented" B3LYP. Calculations on the palladium(II) complexes were performed with these two $x c$ functionals. In case of $\mathrm{c}^{\prime}$ ' the main low energy transition band calculated with M06 consists of two excitations, one is mainly HOMO $\rightarrow$ LUMO, the other is $\mathrm{H}-1 \rightarrow \mathrm{L}+1$ type. CAMB3LYP gives only one excitation, which is a mix of the M06 states. 


\section{Acknowledgements}

This work was partially supported by the European Union and the European Social Fund through project Supercomputer, the national virtual lab (grant no.: TAMOP-4.2.2.C11/1/KONV-2012-0010) (MP).

The research was supported by the EU and co-financed by the European Social Fund under the projects ENVIKUT (TA'MOP-4.2.2.A-11/1/KONV-2012-0043) and TA'MOP 4.2.2/B10/1-2010-0024.

The authors also acknowledge the support of the Hungarian Scientific Research Fund (OTKA K101372 and NK 100857). 


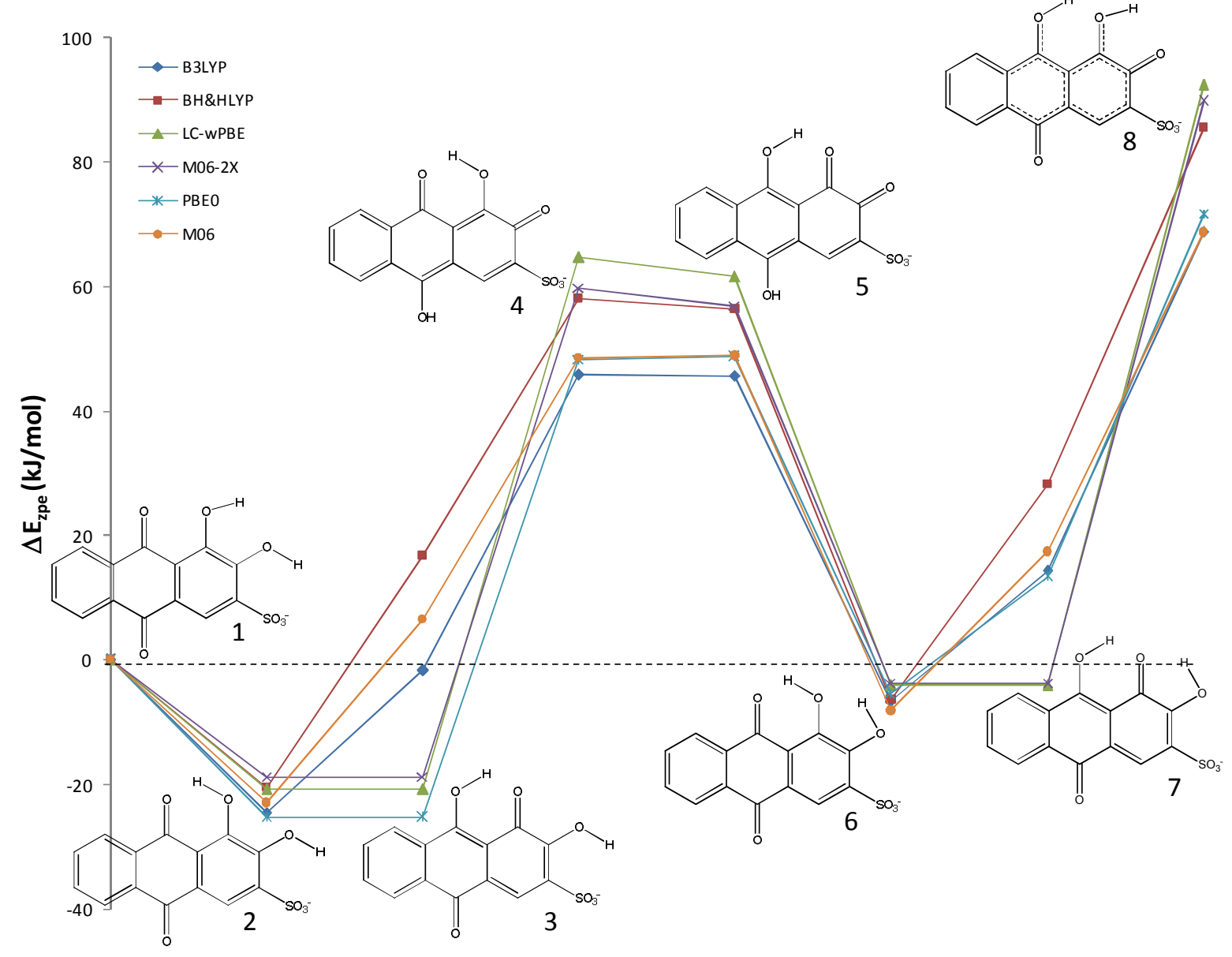

FIG. 1. The possible structures of the monoanionic ARS tautomers $\left(\mathrm{H}_{2} \mathrm{ARS}^{-}\right)$and their relative zero-point corrected electronic energies. The sulpho group is considered to be deprotonated in every calculation. 


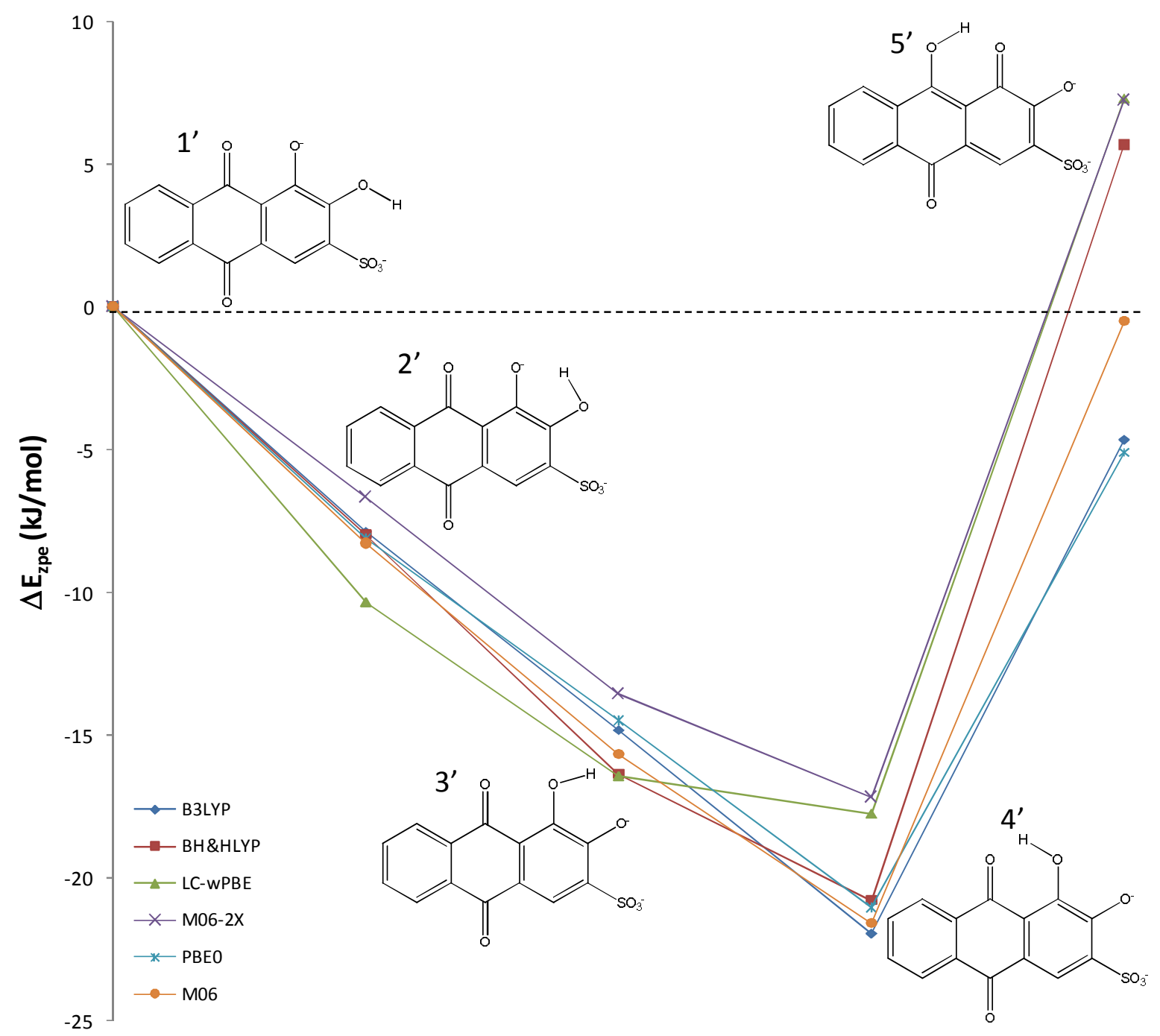

FIG. 2. The structures of the dianionic ARS $\left(\mathrm{HARS}^{2-}\right)$ and their relative zero-point corrected electronic energies. Only those cases were considered where intramolecular hydrogen bonding is possible. 


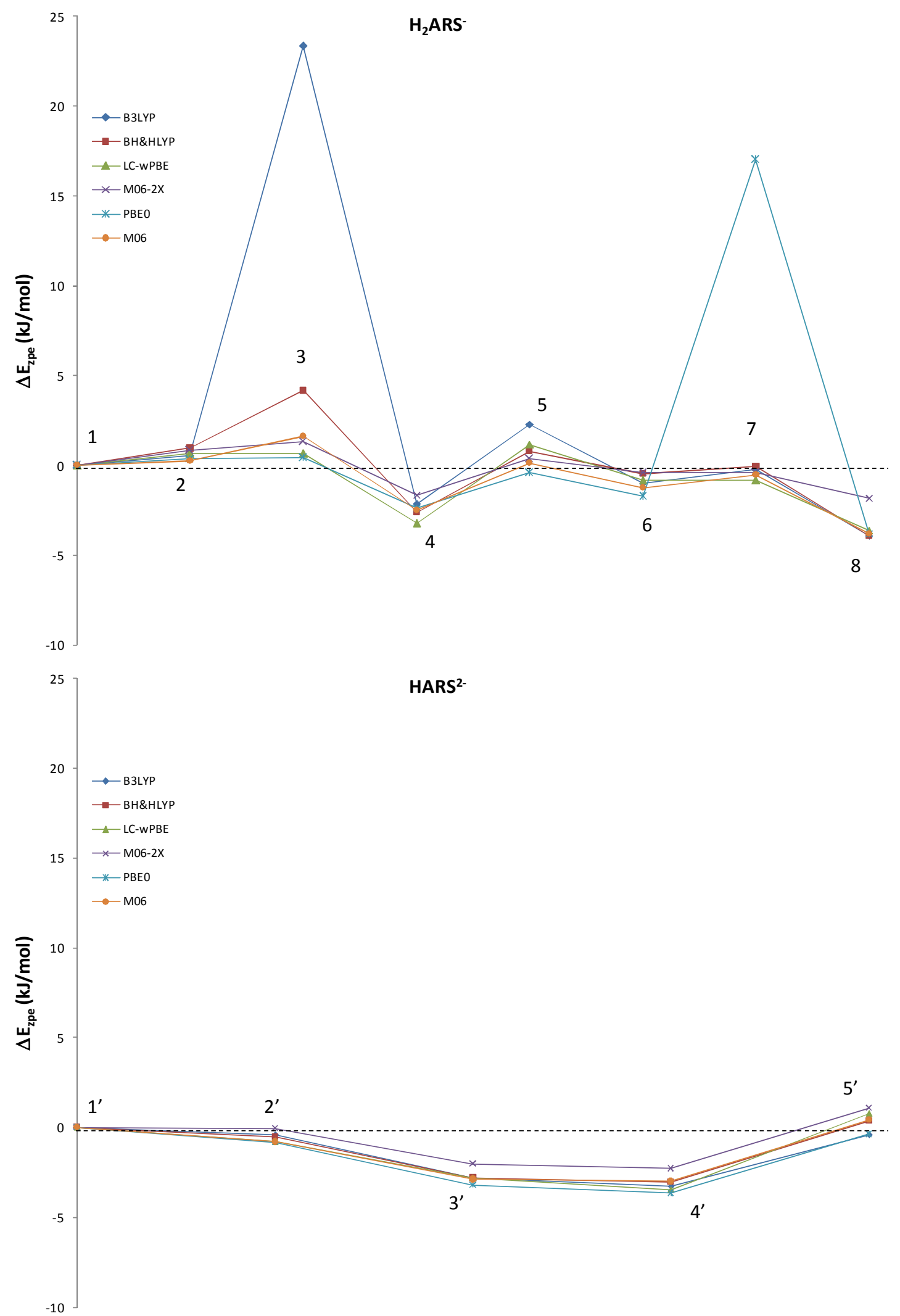

FIG. 3. The difference between the relative energies calculated with $6-311+\mathrm{g}(\mathrm{d}, \mathrm{p})$ and TZVP basis sets. The upper picture shows the monoanionic species, the lower one shows the dianionic forms. 

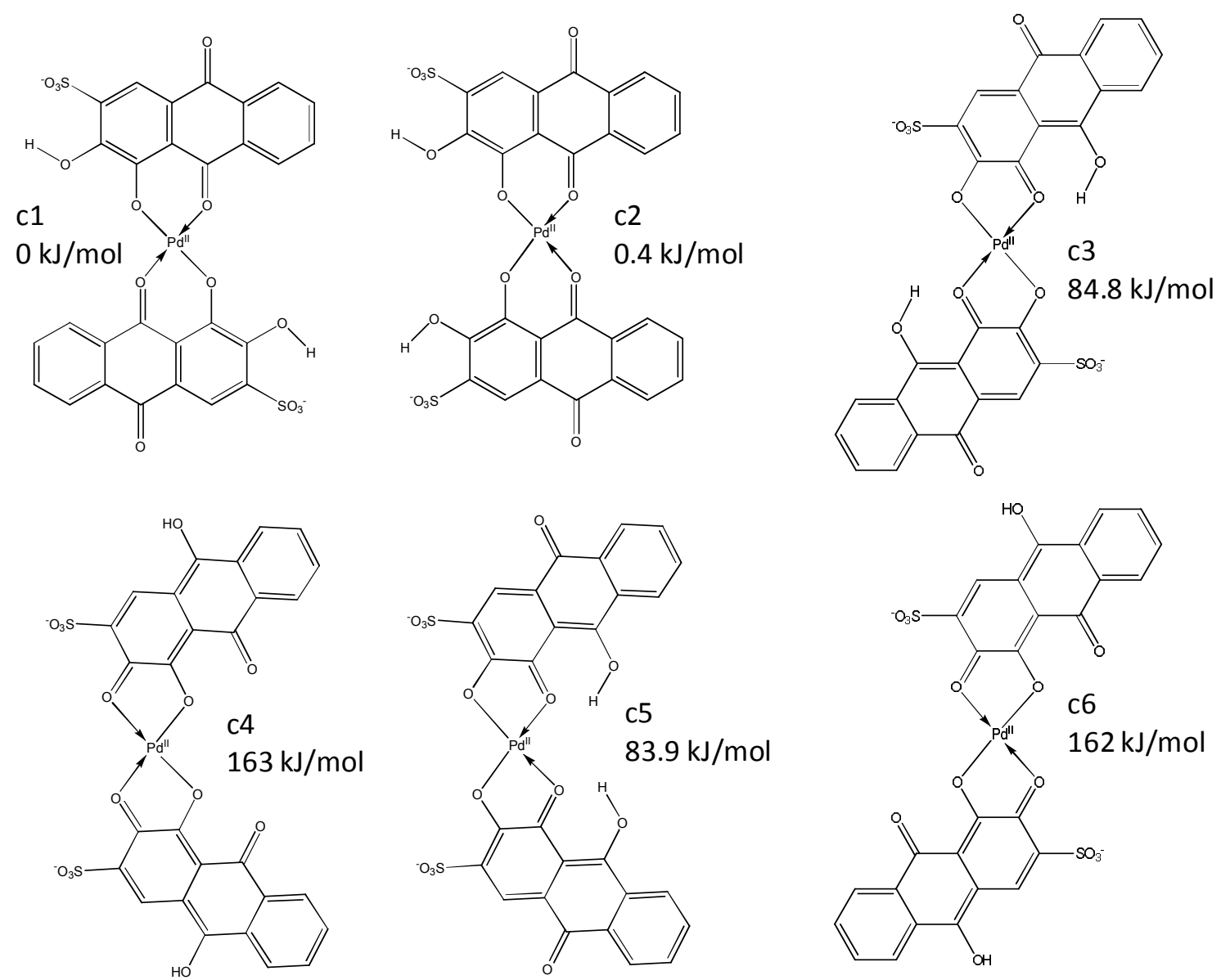

FIG. 4. The structures and relative energies of the studied isomers of the Pd(II)-complexes formed with dianionic ARS ligands. 


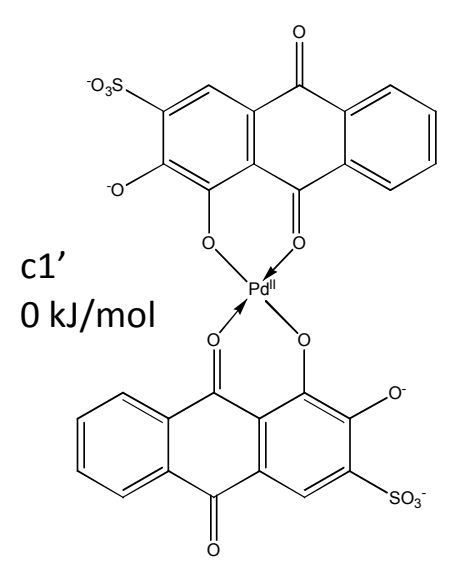

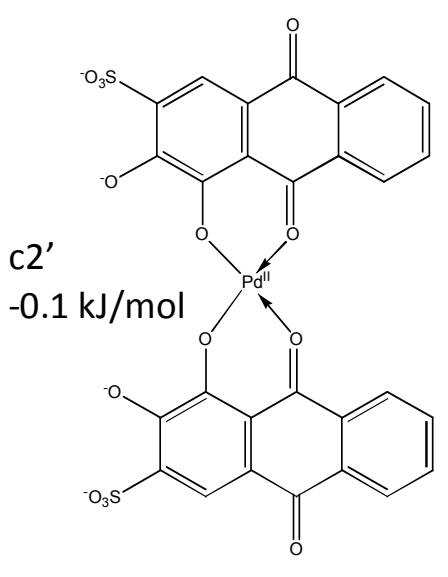
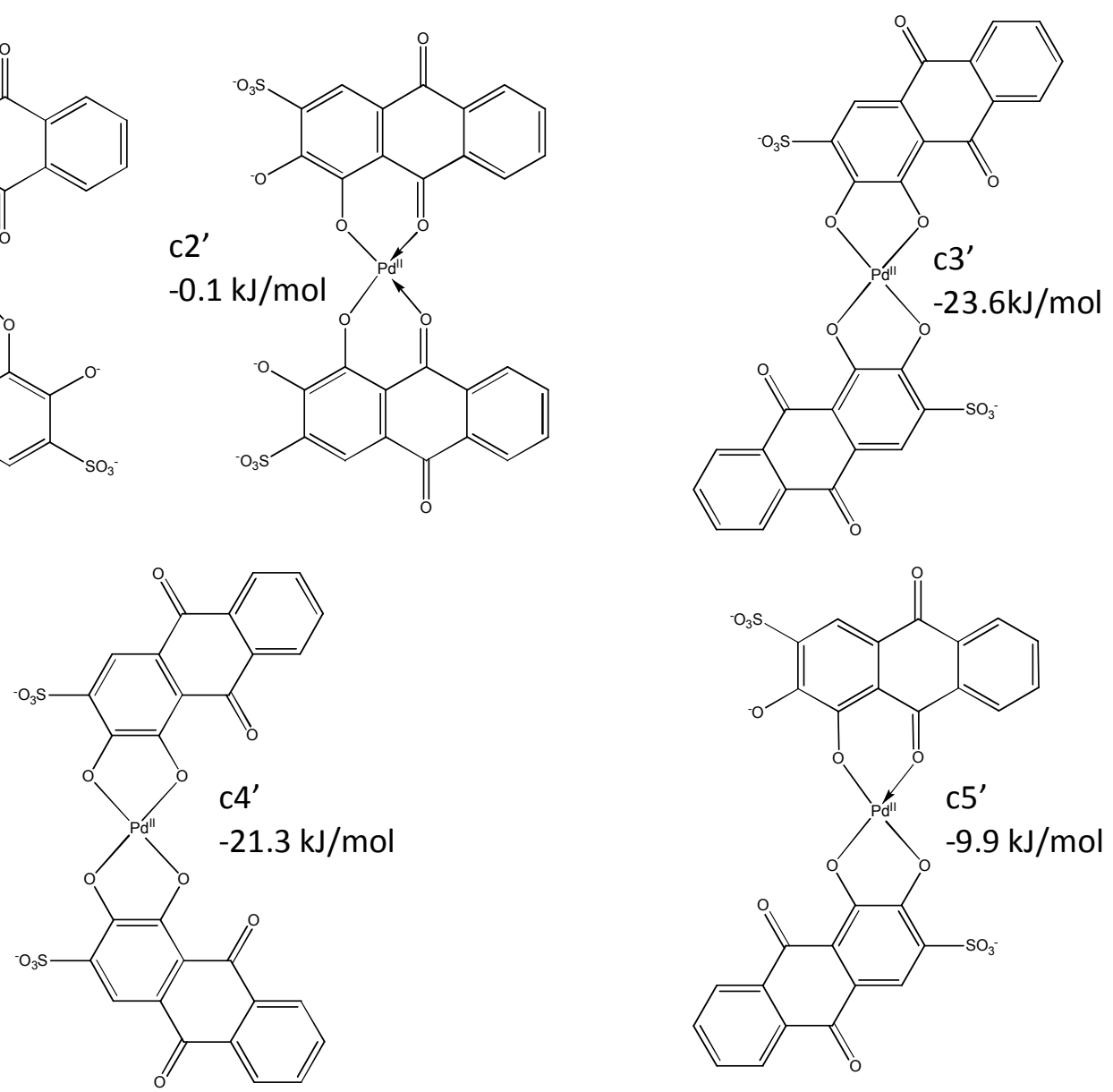

FIG. 5. The structures and relative energies of the studied isomers of the $\mathrm{Pd}(\mathrm{II})$-complexes formed with fully deprotonated ARS ligands. 


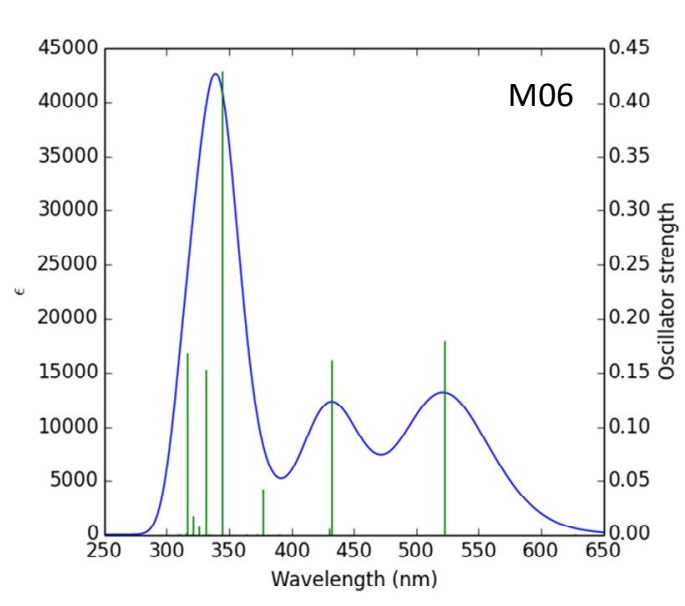

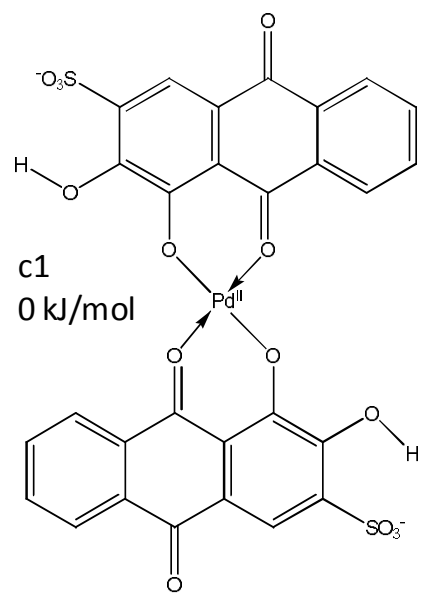

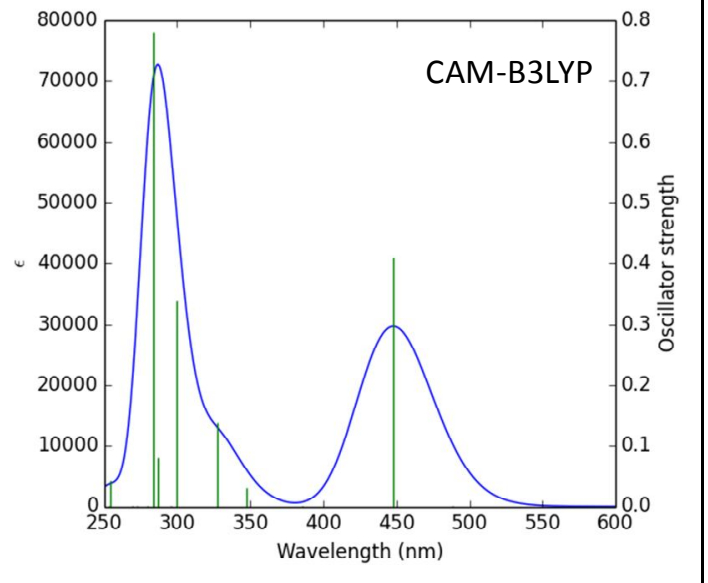

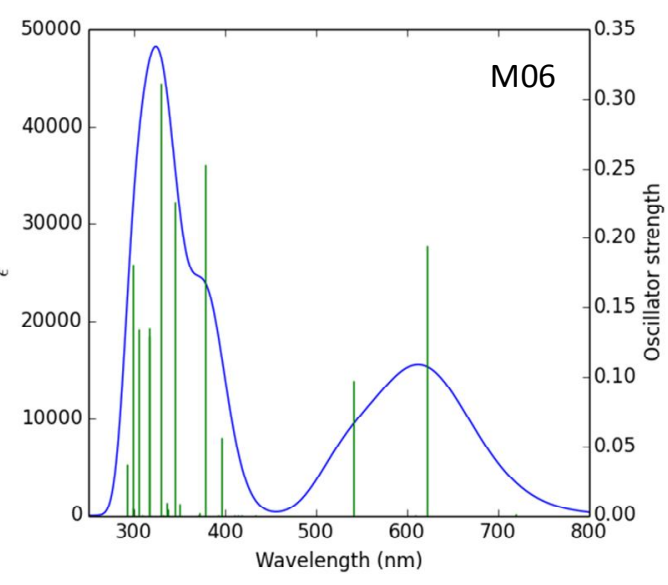
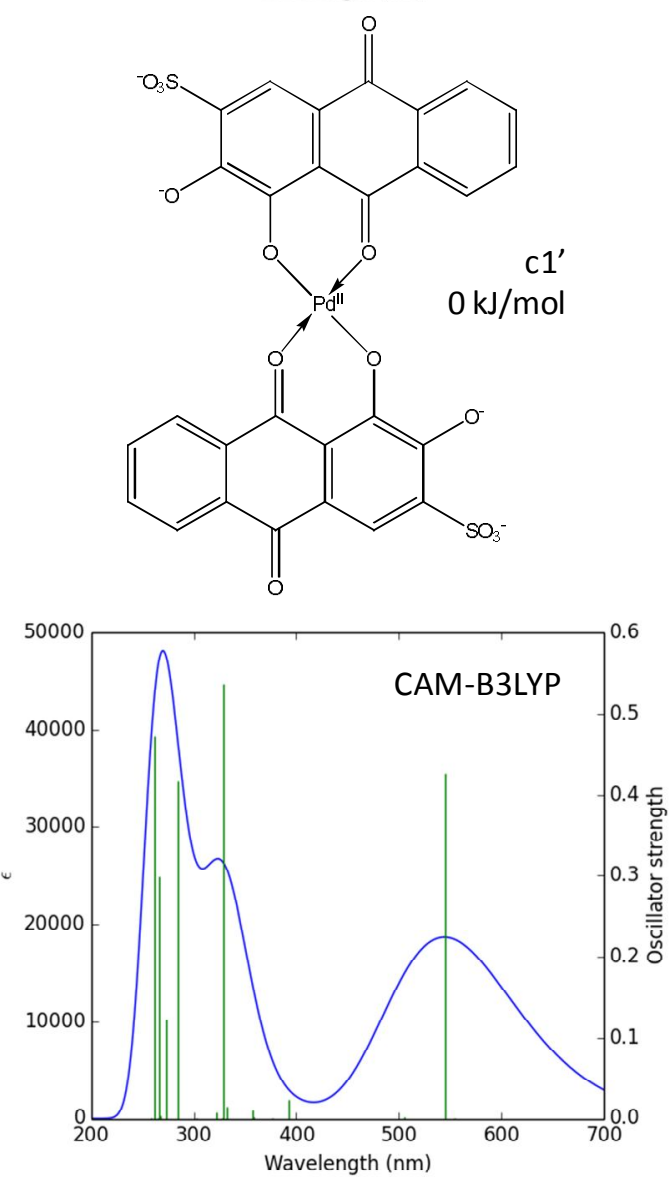

FIG. 6. The simulated UV-Vis spectra and the related structures of the most favorable hydroxy-keto coordinated Pd(II)-ARS complexes. 

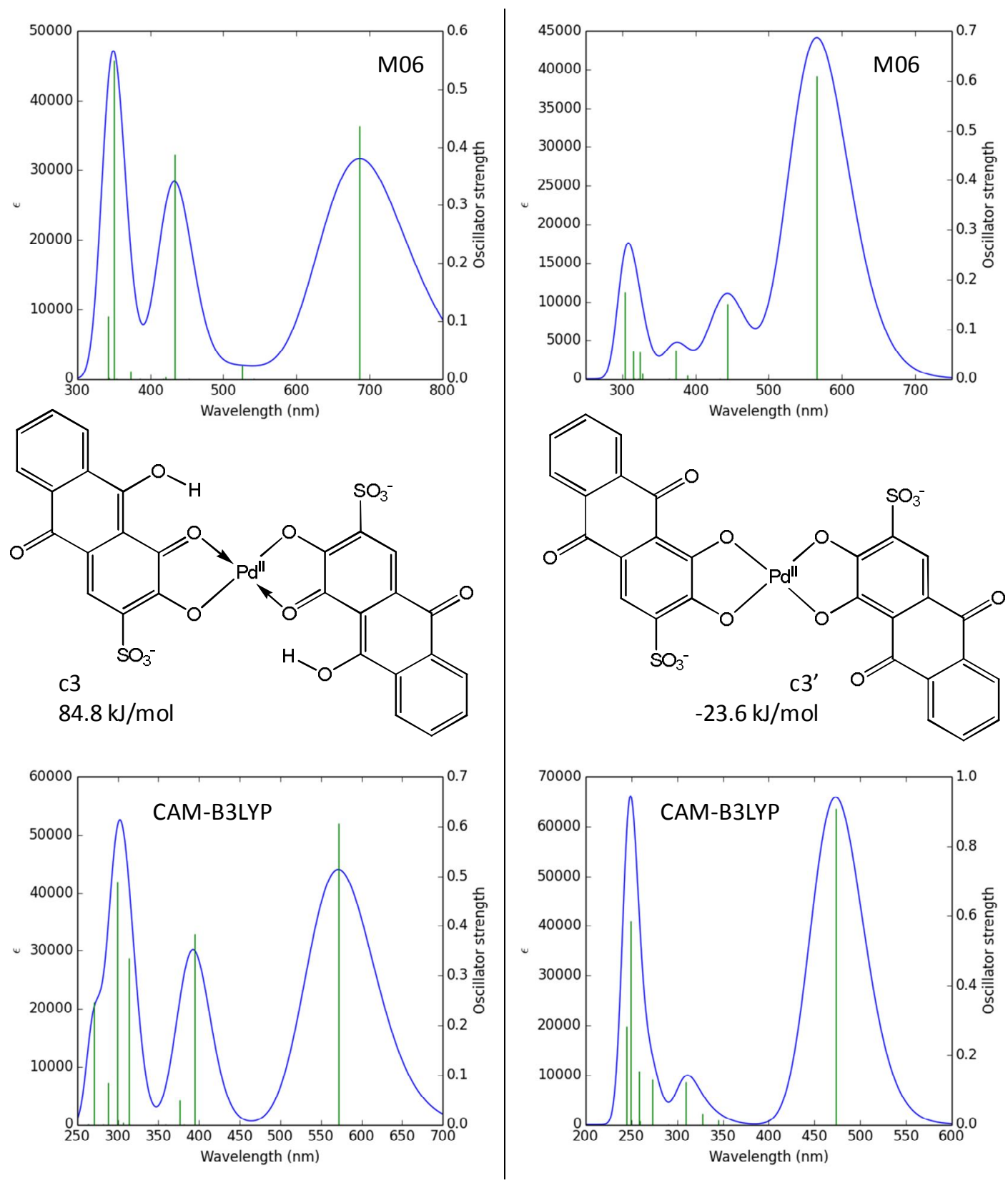

FIG. 7. The simulated UV-Vis spectra and the related structures of the most favorable mono catechol coordinated Pd(II)-ARS complexes. 

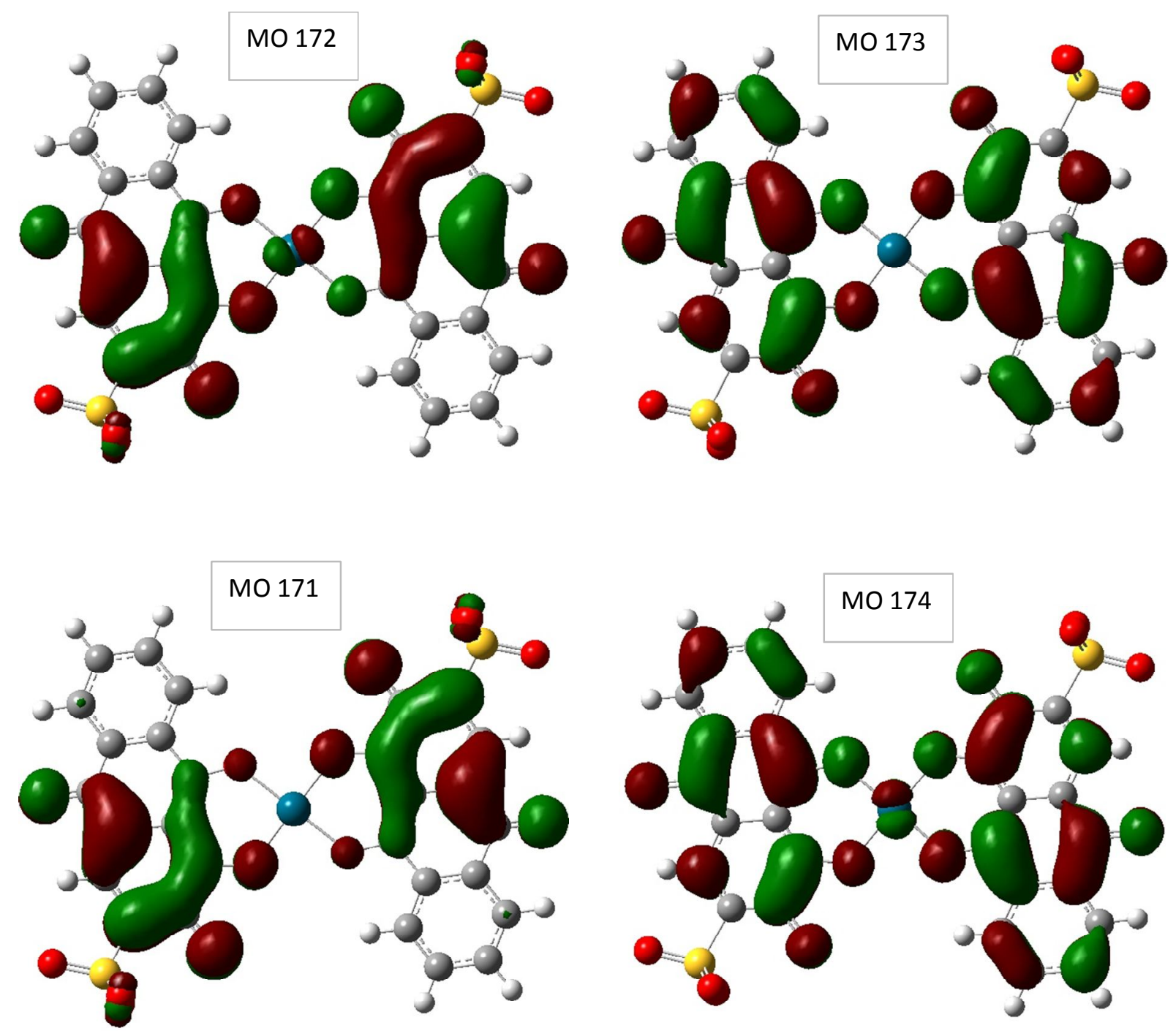

FIG. 8. The molecular orbitals (taken from CAM-B3LYP calculations) of $\mathrm{c} 1$ ' involved in the most important excitations: MO 172 is the HOMO and MO 173 is the LUMO. The main low energy transition is a mix of $172 \rightarrow 173$ and $171 \rightarrow 174$ excitations. 
Table I. Hydrogen bond distances of the dianionic ARS (HARS2`) isomers

\begin{tabular}{|c|c|c|c|c|c|c|}
\hline \multirow{3}{*}{ Functional } & \multicolumn{2}{|c|}{$\begin{array}{c}-\mathrm{O}_{\text {quinone }} \mathrm{H} \cdots \mathrm{O}_{\text {phenolate }} \\
(\mathrm{pm})\end{array}$} & \multicolumn{2}{|c|}{$\begin{array}{c}-\mathrm{O}_{\text {phenolate }} \mathrm{H} \cdots \mathrm{O}_{\text {quinone }} \\
(\mathrm{pm})\end{array}$} & \multicolumn{2}{|c|}{$-\mathrm{O}_{\text {phenolate }} \mathrm{H} \cdots \mathrm{O}_{\text {phenolate }}(\mathrm{pm})$} \\
\hline & \multicolumn{2}{|c|}{ six-membered ring of c 5 , } & \multicolumn{2}{|c|}{ six-membered ring of c4, } & \multicolumn{2}{|c|}{ five-membered ring (c2’; c3') } \\
\hline & $6-311+g(d, p)$ & TZVP & $6-311+g(d, p)$ & TZVP & $6-311+g(d, p)$ & TZVP \\
\hline B3LYP & 152.7 & 151.6 & 163.6 & 162.6 & $180.3 ; 182.1$ & $179.2 ; 180.9$ \\
\hline BH\&HLYP & 160.3 & 159.5 & 168.7 & 167.8 & $183.6 ; 184.8$ & $182.3 ; 183.7$ \\
\hline M06 & 159.9 & 159.2 & 167.6 & 166.8 & $181.6 ; 182.7$ & $180.5 ; 181.8$ \\
\hline M06-2X & 153.6 & 152.0 & 169.3 & 167.9 & $185.8 ; 186.5$ & $184.1 ; 184.9$ \\
\hline PBE0 & 145.2 & 143.4 & 160.4 & 159.1 & $174.4 ; 175.1$ & $173.2 ; 175.6$ \\
\hline LC-wPBE & 150.4 & 149.2 & 165.1 & 164.0 & $174.7 ; 177.0$ & $172.3 ; 175.3$ \\
\hline
\end{tabular}


Table II. Relative errors of different functionals in the calculation of TDDFT excitation energies of different ionic forms of ARS. All calculations were carried out using the IEFPCM model for taking the solvent (water) into account. Experimental values obtained from Ref. 19.

\begin{tabular}{|c|c|c|c|c|c|c|c|}
\hline basis set & b31yp & cam-b3lyp* & $\mathrm{m} 06-2 \mathrm{x}$ & $\mathrm{m} 06$ & BH\&HLYP & PBE0 & LC- $w \mathrm{PBE}$ \\
\hline \multicolumn{8}{|c|}{$\mathrm{ARS}^{3-}\left(\exp . \lambda_{\max }=514 \mathrm{~nm}\right)$} \\
\hline $6-311+g(d, p)$ & $11.1 \%$ & $-0.4 \%$ & $-1.4 \%$ & $6.8 \%$ & $-5.8 \%$ & $7.6 \%$ & $-11.7 \%$ \\
\hline TZVP & $9.9 \%$ & - & $-2.7 \%$ & $6.0 \%$ & $-7.0 \%$ & $6.6 \%$ & $-12.6 \%$ \\
\hline \multicolumn{8}{|c|}{$\mathrm{HARS}^{2-}\left(\exp . \lambda_{\max }=510\right.$ and $\left.326 \mathrm{~nm}\right)$} \\
\hline \multirow{2}{*}{$6-311+g(d, p)$} & $15.5 \%$ & $-3.5 \%$ & $-6.7 \%$ & $8.0 \%$ & $-10.4 \%$ & $10.4 \%$ & $-17.8 \%$ \\
\hline & $12.3 \%$ & $-7.7 \%$ & $-6.7 \%$ & $7.4 \%$ & $-* *$ & $8.0 \%$ & $-* *$ \\
\hline \multirow{2}{*}{ TZVP } & $15.7 \%$ & - & $-7.1 \%$ & $7.8 \%$ & $-10.8 \%$ & $10.6 \%$ & $-18.0 \%$ \\
\hline & $11.7 \%$ & - & $-7.7 \%$ & $6.4 \%$ & $-* *$ & $7.7 \%$ & $-* *$ \\
\hline \multicolumn{8}{|c|}{$\mathrm{H}_{2} \mathrm{ARS}^{-}\left(\exp . \lambda_{\max }=425 \mathrm{~nm}\right)$} \\
\hline $6-311+g(d, p)$ & $4.7 \%$ & $-11.1 \%$ & $-15.8 \%$ & $-0.9 \%$ & $-17.9 \%$ & $0.7 \%$ & $-22.1 \%$ \\
\hline TZVP & $4.7 \%$ & - & $-16.0 \%$ & $-1.4 \%$ & $-18.4 \%$ & $0.7 \%$ & $-22.4 \%$ \\
\hline Average: & $10.7 \%$ & $-5.7 \%$ & $-8.0 \%$ & $5.0 \%$ & $-11.7 \%$ & $6.5 \%$ & $-17.4 \%$ \\
\hline
\end{tabular}

*Geometry optimizations were done at b3lyp/6-311+g(d,p) level of theory

**Only one excitation calculated above $300 \mathrm{~nm}$ (only two peaks in the calculated spectra) 
[1] A.R. Eberle, M.W. Lerner, Separation and Determination of Scandium Spectrophotometric Method Using Alizarin Red S, Analytical Chemistry, 27 (1955) 15511554.

[2] S. Natelson, R. Penniall, Colorimetric Estimation of Ultramicro Quantities of Calcium in Human Serum as Complex with Alizarin, Analytical Chemistry, 27 (1955) 434-437.

[3] C.A. Gregory, W. Grady Gunn, A. Peister, D.J. Prockop, An Alizarin red-based assay of mineralization by adherent cells in culture: comparison with cetylpyridinium chloride extraction, Analytical Biochemistry, 329 (2004) 77-84.

[4] Y.A. Rozin, L.V. Tat'yanenko, E.I. Buryndina, A.S. Barybin, V.G. Popova, Alizarin derivatives as inhibitors of calcium transport, Pharm Chem J, 30 (1996) 520-522.

[5] Y. Kubo, T. Ishida, A. Kobayashi, T.D. James, Fluorescent alizarin-phenylboronic acid ensembles: design of self-organized molecular sensors for metal ions and anions, Journal of Materials Chemistry, 15 (2005) 2889-2895.

[6] C.O. Sreekala, P. Balraju, Y.S. Deol, P. Pradeep, M.S. Roy, Photo Response in Zinc Oxide Doped Alizarin Thin Film, AIP Conference Proceedings, 1004 (2008) 230-234.

[7] S. Schumacher, T. Nagel, F.W. Scheller, N. Gajovic-Eichelmann, Alizarin Red S as an electrochemical indicator for saccharide recognition, Electrochimica Acta, 56 (2011) 66076611.

[8] Y.-Y. Jiang, K. Wang, C.-Z. Xu, X.-D. Yang, H.-H. Li, Application of Alizarin/GrapheneChitosan Modified Electrode on Detection of Human Telomere DNA, Chinese Journal of Analytical Chemistry, 41 (2013) 481-487.

[9] D. Jacquemin, E.A. Perpète, G.E. Scuseria, I. Ciofini, C. Adamo, TD-DFT Performance for the Visible Absorption Spectra of Organic Dyes: Conventional versus Long-Range Hybrids, Journal of Chemical Theory and Computation, 4 (2007) 123-135.

[10] M. Savko, S. Kaščáková, P. Gbur, P. Miškovský, J. Uličný, Performance of Time Dependent Density Functional Theory on excitations of medium sized molecules - Test on ionic forms of anthraquinone dihydroxy derivatives, Journal of Molecular Structure: THEOCHEM, 823 (2007) 78-86.

[11] J. Preat, A.D. Laurent, C. Michaux, E.A. Perpète, D. Jacquemin, Impact of tautomers on the absorption spectra of neutral and anionic alizarin and quinizarin dyes, Journal of Molecular Structure: THEOCHEM, 901 (2009) 24-30.

[12] P. Dev, S. Agrawal, N.J. English, Determining the appropriate exchange-correlation functional for time-dependent density functional theory studies of charge-transfer excitations in organic dyes, The Journal of Chemical Physics, 136 (2012) -

[13] A. Le Person, J.-P. Cornard, S. Say-Liang-Fat, Studies of the tautomeric forms of alizarin in the ground state by electronic spectroscopy combined with quantum chemical calculations, Chemical Physics Letters, 517 (2011) 41-45.

[14] D.H. Douma, B. M'Passi-Mabiala, R. Gebauer, Optical properties of an organic dye from time-dependent density functional theory with explicit solvent: The case of alizarin, The Journal of Chemical Physics, 137 (2012) -.

[15] J. Mech, M.A. Grela, K. Szaciłowski, Ground and excited state properties of alizarin and its isomers, Dyes and Pigments, 103 (2014) 202-213.

[16] M. Ghaedi, A. Hassanzadeh, S.N. Kokhdan, Multiwalled Carbon Nanotubes as

Adsorbents for the Kinetic and Equilibrium Study of the Removal of Alizarin Red S and Morin, Journal of Chemical \& Engineering Data, 56 (2011) 2511-2520. 
[17] D. Wang, S.A. Bradford, R.W. Harvey, B. Gao, L. Cang, D. Zhou, Humic Acid Facilitates the Transport of ARS-Labeled Hydroxyapatite Nanoparticles in Iron Oxyhydroxide-Coated Sand, Environmental Science \& Technology, 46 (2012) 2738-2745.

[18] Z. Wang, X. Liu, W.R.G. Baeyens, J.R. Delanghe, J. Ouyang, Copper(II)-Alizarin Red S Complex as an Efficient Chemiluminescent Probe for the Detection of Human Serum Proteins after Polyacrylamide Gel Electrophoresis, Journal of Proteome Research, 7 (2008) 50755081.

[19] O. Thomas, C. Burgess, UV-visible Spectrophotometry of Water and Wastewater, Elsevier, Amsterdam, 2007.

[20] D. Jacquemin, J. Preat, M. Charlot, V. Wathelet, J.-M. André, E.A. Perpète, Theoretical investigation of substituted anthraquinone dyes, The Journal of Chemical Physics, 121 (2004) 1736-1743.

[21] A.D. Laurent, D. Jacquemin, TD-DFT benchmarks: A review, International Journal of Quantum Chemistry, 113 (2013) 2019-2039.

[22] M.J. Frisch, G.W. Trucks, G.E.S. H. B. Schlegel, M. A. Robb, J. R. Cheeseman, G. Scalmani, V. Barone, B. Mennucci, G. A. Petersson, H. Nakatsuji, M. Caricato, X. Li, H. P. Hratchian, A. F. Izmaylov, J. Bloino, G. Zheng, J. L. Sonnenberg, M. Hada, M. Ehara, K. Toyota, R. Fukuda, J. Hasegawa, M. Ishida, T. Nakajima, Y. Honda, O. Kitao, H. Nakai, T. Vreven, J. A. Montgomery, Jr., J. E. Peralta, F. Ogliaro, M. Bearpark, J. J. Heyd, E. Brothers, K. N. Kudin, V. N. Staroverov, R. Kobayashi, J. Normand, K. Raghavachari, A. Rendell, J. C. Burant, S. S. Iyengar, J. Tomasi, M. Cossi, N. Rega, J. M. Millam, M. Klene, J. E. Knox, J. B. Cross, V. Bakken, C. Adamo, J. Jaramillo, R. Gomperts, R. E. Stratmann, O. Yazyev, A. J. Austin, R. Cammi, C. Pomelli, J. W. Ochterski, R. L. Martin, K. Morokuma, V. G. Zakrzewski, G. A. Voth, P. Salvador, J. J. Dannenberg, S. Dapprich, A. D. Daniels, Ö. Farkas, J. B. Foresman, J. V. Ortiz, J. Cioslowski, and D. J. Fox, Gaussian 09, Gaussian, Inc, Wallingford CT, 2009.

[23] Y. Zhao, D. Truhlar, The M06 suite of density functionals for main group thermochemistry, thermochemical kinetics, noncovalent interactions, excited states, and transition elements: two new functionals and systematic testing of four M06-class functionals and 12 other functionals, Theor Chem Account, 120 (2008) 215-241.

[24] M.L. Crossley, GENTIAN VIOLET-ITS SELECTIVE BACTERICIDAL ACTION.1, Journal of the American Chemical Society, 41 (1919) 2083-2090.

[25] V.Y. Fain, B.E. Zaitsev, M.A. Ryabov, Metal Complexes with Alizarin and Alizarin Red S: Electronic Absorption Spectra and Structure of Ligands, Russian Journal of Coordination Chemistry, 30 (2004) 365-370.

[26] M. Doskocz, K. Kubas, A. Frąckowiak, R. Gancarz, NMR and ab initio studies of Mg2+, $\mathrm{Ca} 2+, \mathrm{Zn2+}$, Cu2+ alizarin complexes, Polyhedron, 28 (2009) 2201-2205.

[27] F. Joó, N. Balogh, L. Horváth, G. Filep, I. Horváth, L. Vígh, Complex hydrogenation/oxidation reactions of the water-soluble hydrogenation catalyst palladium di (sodium alizarinmonosulfonate) and details of homogeneous hydrogenation of lipids in isolated biomembranes and living cells, Analytical Biochemistry, 194 (1991) 34-40.

[28] A.V. Bulatov, A.T. Nikitaev, M.L. Khidekel, Radical intermediates in hydrogenation of aromatic nitro compounds in presence of platinum(ii) complex with alizarin, Russ Chem Bull, 30 (1981) 704-705.

[29] N.M. O'Boyle, A.L. Tenderholt, K.M. Langner, cclib: A library for package-independent computational chemistry algorithms, Journal of Computational Chemistry, 29 (2008) 839845. 
[30] S. Miertuš, E. Scrocco, J. Tomasi, Electrostatic interaction of a solute with a continuum. A direct utilizaion of $A B$ initio molecular potentials for the prevision of solvent effects, Chemical Physics, 55 (1981) 117-129.

[31] A.D. Becke, Density-functional thermochemistry. III. The role of exact exchange, The Journal of Chemical Physics, 98 (1993) 5648-5652.

[32] A.D. Becke, A new mixing of Hartree-Fock and local density-functional theories, The Journal of Chemical Physics, 98 (1993) 1372-1377.

[33] C. Adamo, V. Barone, Toward reliable density functional methods without adjustable parameters: The PBE0 model, The Journal of Chemical Physics, 110 (1999) 6158-6170.

[34] Y. Tawada, T. Tsuneda, S. Yanagisawa, T. Yanai, K. Hirao, A long-range-corrected timedependent density functional theory, The Journal of Chemical Physics, 120 (2004) 84258433.

[35] O.A. Vydrov, G.E. Scuseria, Assessment of a long-range corrected hybrid functional, The Journal of Chemical Physics, 125 (2006) -.

[36] T. Yanai, D.P. Tew, N.C. Handy, A new hybrid exchange-correlation functional using the Coulomb-attenuating method (CAM-B3LYP), Chemical Physics Letters, 393 (2004) 51-57.

[37] A.D. McLean, G.S. Chandler, Contracted Gaussian basis sets for molecular calculations. I. Second row atoms, Z=11-18, The Journal of Chemical Physics, 72 (1980) 5639-5648.

[38] A. Schäfer, C. Huber, R. Ahlrichs, Fully optimized contracted Gaussian basis sets of triple zeta valence quality for atoms Li to Kr, The Journal of Chemical Physics, 100 (1994) 58295835.

[39] L.A. LaJohn, P.A. Christiansen, R.B. Ross, T. Atashroo, W.C. Ermler, Abinitio relativistic effective potentials with spin-orbit operators. III. Rb through Xe, The Journal of Chemical Physics, 87 (1987) 2812-2824.

[40] M.I. Purgel, M. Maliarik, J. Glaser, C. Platas-Iglesias, I. Persson, I. Tóth, Binuclear Pt-TI Bonded Complex with Square Pyramidal Coordination around Pt: A Combined Multinuclear NMR, EXAFS, UV-Vis, and DFT/TDDFT Study in Dimethylsulfoxide Solution, Inorganic Chemistry, 50 (2011) 6163-6173. 